POS $\quad \begin{aligned} & \text { PROCEEDINGS } \\ & \text { OF SCIENCE }\end{aligned}$

\title{
The 5th International Workshop on Deep Learning in Computational Physics - DLCP-2021
}

\section{Andreas Haungs ${ }^{a, *}$ and Alexander Kryukov ${ }^{b}$}

${ }^{a}$ Karlsruhe Institute of Technology, Institute for Astroparticle Physics, Karlsruhe, Germany

${ }^{b}$ Lomonosov Moscow State University, Skobeltsyn Institute of Nuclear Physics, Moscow, Russia

E-mail: andreas.haungs@kit.edu, kryukov@theory.sinp.msu.ru

Preface to the $5^{\text {th }}$ International Workshop on Deep Learning in Computational Physics (DLCP-2021) hold online on 28-29 June, 2021.

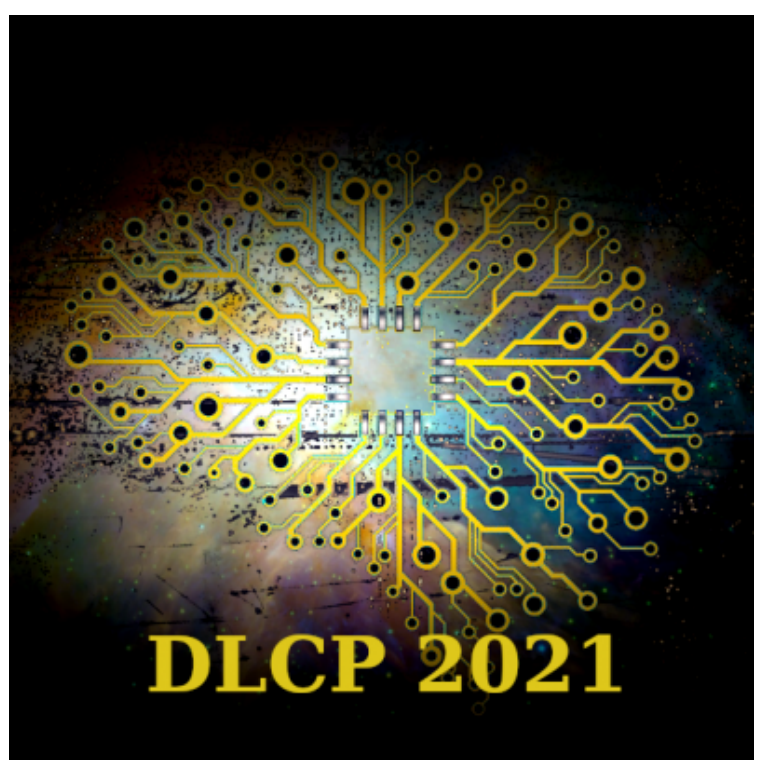

The 5th International Workshop on Deep Learning in Computational Physics 28-29 June, 2021

Moscow, Russia

${ }^{*}$ Speaker 
The international workshop DLCP-2021, hold online on June 28th and 29th, 2021, was dedicated on "Deep Learning in Computational Physics".

The workshop primarily focuses on the use of machine learning in cosmic-ray astrophysics, but was not limited to this area. Topics of interest are various applications of artificial neural networks to physical problems, as well as the development of new modern machine learning methods for analysing various scientific data, including big data.

Modern large-scale physics experiments generate a huge data stream, measured in at least hundreds of terabytes. The time of their active operation can reach several decades, and the amount of accumulated data can exceed one hundred petabytes. In this context, the issue of active and on-going management of data as well as modern and sophisticated analyses methods throughout their life cycle is a very important and highly topical issue.

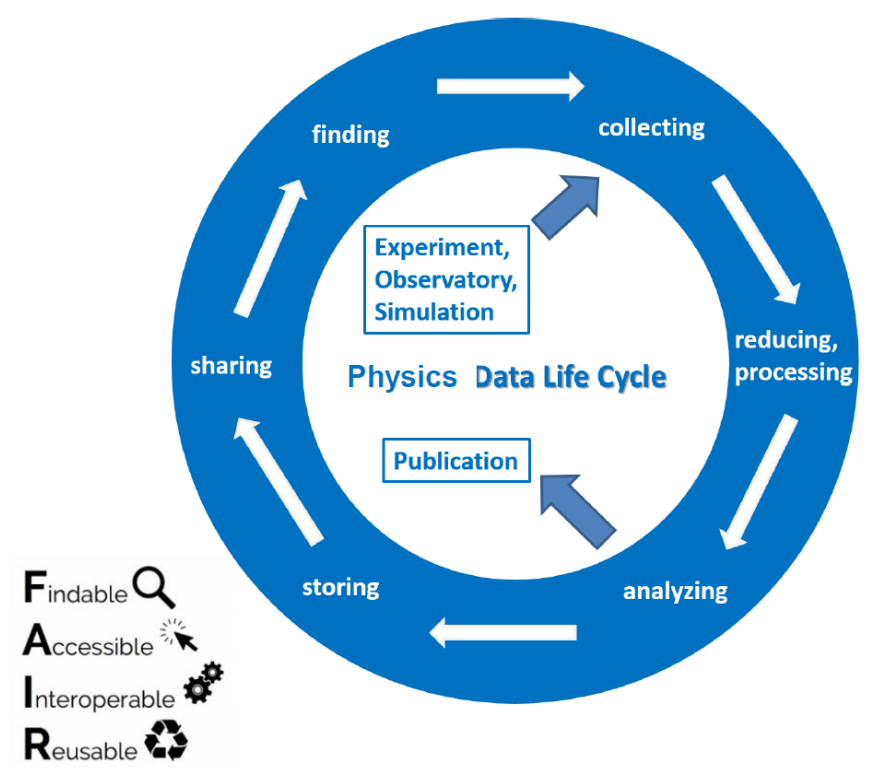

Figure 1: General data life cycle scheme in physics, where the DLCP-2021 was dedicated to the development of modern analysis methods. For a useful and efficient life cycle each step must be based on a FAIR data and metadata treatment.

This workshop is part of a cooperation between Russia and Germany, funded by Helmholtz and RSF, dedicated to the digitisation of astroparticle physics [1,2]. The baseline approach of this cooperation is to establish FAIR Data Life Cycle Concepts and Open Data in the research field. A typical Data Life Cycle is shown in Fig. 1. DLCP-2021 was dedicated to two stages of data life cycle: (i) processing data and (ii) analysing data. These are key items for the success of any modern experiment.

Currently, methods of data processing and data analysis based on machine learning are actively developing in various fields of science and technology. These methods have shown outstanding results in the field of natural language text analysis, face recognition, social network analysis, and so 
on. These methods have become more and more applied in natural sciences as physics, chemistry, medicine, in problems of climate modelling or in engineering sciences. Therefore, the DLCP-2021 workshop was devoted to machine learning methods for processing and analysing data from scientific experiments. In particular, special attention has been paid to problems in astroparticle physics.

The current workshop was attended by 32 scientists from Russia and Germany as well as from other countries. In total, the scientific editor board selected 17 articles for publication, which present the results of original research.

\section{References}

[1] Proceedings of the 3rd International Workshop on Data Life Cycle in Physics (DLCP-2019), Irkutsk, Russia , April 2-7, 2019. Edited by: Alexander Kryukov, Andreas Haungs; CEURWS: 12 Jul 2019, http: //ceur-ws . org/Vol-2406/

[2] Proceedings of the 4th International Workshop on Data Life Cycle in Physics (DLCP-2020), Moscow, Russia, June 8-10, 2020. Edited by: Alexander Kryukov, Andreas Haungs; CEURWS: 21 Sep 2020, http: //ceur-ws . org/Vol-2679/ 